\title{
CASE OF CONGENITAL ATRESIA OF THE OESOPHAGUS WITH INFANTILE PYLORIC STENOSIS
}

\author{
BY \\ G. E. FOSTER, Major, R.A.M.C. \\ and \\ R. E. SHAW, M.D. \\ Pathologist, King Edward VII Memorial Hospital, Bermuda
}

History. A healthy white woman of 24 was delivered on October 14, 1944, of her third full term male child which weighed $5 \mathrm{lb} .15 \mathrm{oz}$. The infant cried promptly but was cyanosed. There was abundant mucus in the pharynx, and moist medium râles were present throughout the chest. No pyloric tumour was noted. Oxygen by nasal funnel relieved the cyanosis until the child fed, whereupon, within twenty seconds, it became deeply cyanotic, choked, and regurgitated nearly all the feeding. Hypodermoclysis with 5 per cent. glucose saline was instituted.

X-ray investigation was carried out on October 17, 1944.

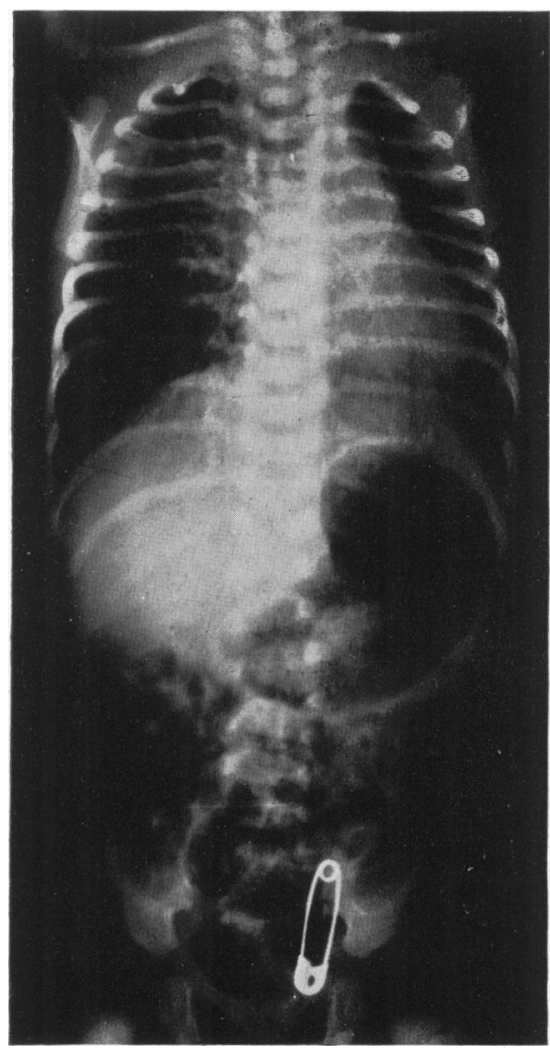

FIG. 1, A.-X-ray of thorax and abdomen before ingestion of barium.
1. FLUOROSCOPY. Thin barium suspension was given under the fluoroscope and was seen to fill the oesophagus to the level of the clavicle, where it collected as in a pouch, which when full contracted and expelled the barium.

2. FILMS of the thorax and abdomen after barium (fig. 1, B) show that the bronchi are outlined, and fine mottling is seen in the right and left lung fields.

Those of the abdomen show that the stomach contains gas and, in its distal portion, a dense shadow such as that caused by barium.

3. IMPRESSION. Congenital atresia of the oesophagus with tracheo-oesophageal fistula.

Progress. After consultation with the senior

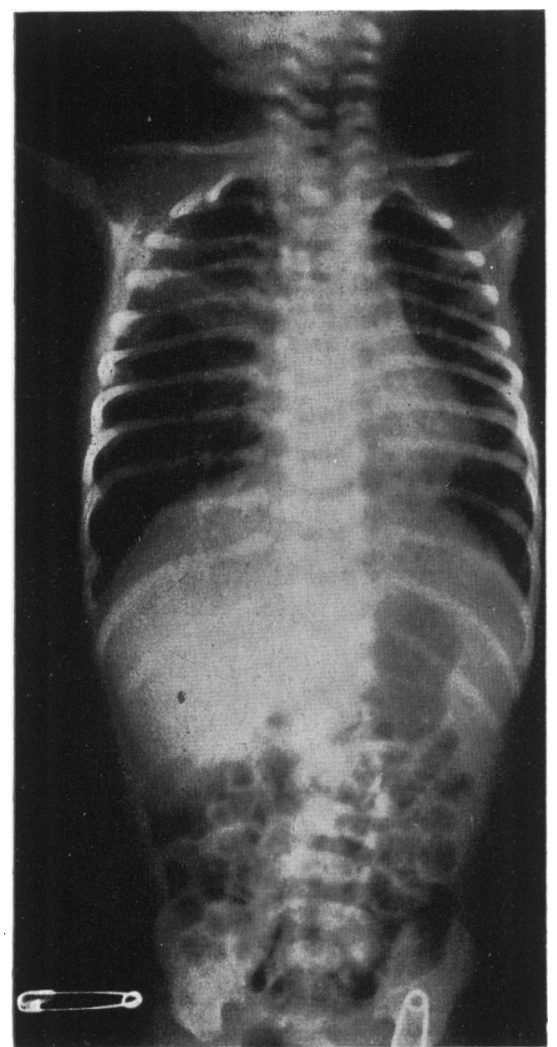

Fig. 1, B.-X-ray after ingestion of barium. (a) Outlined bronchi. (b) Fine mottling both lung fields. (c) Barium shadow in stomach. 


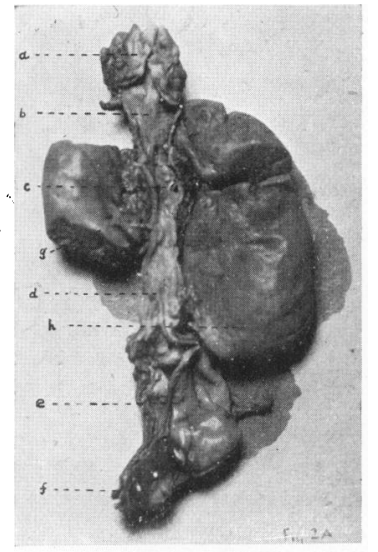

A

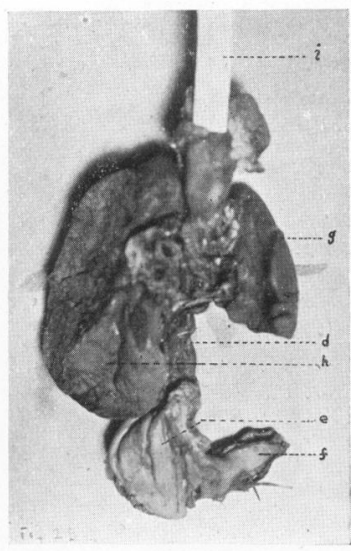

B

FIG. 2.-Photograph of specimen.
(A) Anterior aspect.
(B) Posterior aspect.

(a) Larynx. (b) Trachea. (c) Bifurcation of trachea. (d) Distal oesophagus. (e) Stomach. ( $f$ ) Pylorus (hypertrophic, stenosed). ( $g$ ) Right lung (lower portion cut away). (h) Left lung. (i) Director in oesophageal pouch (proximal oesophagus).

Bermuda surgeon, Mr. W. E. Tucker, surgery was considered inadvisable. The child died on the eighth day.

Necropsy report (Dr. R. E. Shaw). The body was that of an emaciated white male infant, aged eight days, weighing $4 \frac{1}{2} \mathrm{lb}$.

The stomach, oesophagus, lungs, trachea, larynx, heart, and great vessels were removed en masse. On opening the stomach and lower oesophagus, the latter was found to join the trachea posteriorly at its bifurcation. The trachea was otherwise normal, except that the mucous membrane had a more linear appearance where it opened into the oesophagus. The proximal portion of the oesophagus ended in a blind pouch one inch below the level of the cricoid cartilages of the larynx (fig. 2).

The lungs presented a mottled appearance, the mottling consisting of red, firm areas, some of which appeared as atelectatic spots while others were pneumonic; these red areas were scattered throughout both lungs.

The stomach was normal except at its pyloric end, where there was hypertrophy of the pylorus with consequent stenosis.

The bowel was shrunken and contained only a small amount of fluid and mucus.

The liver, pancreas, spleen, genito-urinary and osseous systems, were normal.

Summary. Death was due to inanition and pneumonia due to congenital atresia of the oesophagus with tracheo-oesophageal fistula. Infantile pyloric stenosis was present.

\section{Discussion}

1. Grey Turner stated (1944):

(i) Congenital atresia of the oesophagus occurs once in about 2500 births;

(ii) Four out of 21 such cases operated upon by Cameron Haight in America recovered;

(iii) Operation should be performed on the first or second day of life.

2. The association of infantile pyloric stenosis with congenital atresia of the oesophagus, unless this is merely a coincidence, is important if surgical treatment of the latter is considered, since, if unrecognized, it might vitiate a successful operation.

3. The early onset of infantile pyloric stenosis in this case may possibly be related to the absence of food in the stomach, with consequent non-neutralization of the gastric juices (Engel's theory of hyperacidity).

\section{R EFERENCE}

Turner, G. (1944). Newc. med. J., 22, 1. 